\title{
Parcellations and Connectivity Patterns in Human and Macaque Cerebral Cortex
}

\author{
David C. Van Essen, Chad Donahue, Donna L. Dierker, \\ and Matthew F. Glasser
}

\begin{abstract}
To decipher brain function, it is vital to know how the brain is wired. This entails elucidation of brain circuits at multiple scales, including microscopic, mesoscopic, and macroscopic levels. Here, we review recent progress in mapping the macroscopic brain circuits and functional organization of the cerebral cortex in primates-humans and macaque monkeys, in particular. There are many similarities across species in terms of overall patterns of cortical gray matter myelination as well as functional areas that are presumed to be homologous. However, there are also many important species differences, including cortical convolutions that are much more complex and more variable in humans than in monkeys. Our ability to analyze structure and function has benefited from improved methods for intersubject registration that cope with this individual variability. To characterize long-distance connectivity, powerful but indirect methods are now available, including resting-state functional connectivity and diffusion imaging coupled with probabilistic tractography. We illustrate how connectivity inferred from diffusion imaging and tractography can be evaluated in relation to 'ground truth' based on anatomical tracers in the macaque. Interspecies registration between human and macaque cortex based on presumed interspecies homologies demonstrates an impressive degree of areal expansion in regions associated with higher cognitive function.
\end{abstract}

\section{Introduction}

The past decade has seen an explosion of interest and information about mammalian brain connectivity over a wide range of spatial scales, including the macroscopic, microscopic, and mesoscopic levels discussed in the present volume. For the human brain, there has been exciting progress in examining brain connectivity and function using increasingly powerful methods of noninvasive imaging. These

D.C. Van Essen $(\varangle) \cdot$ C. Donahue • D.L. Dierker • M.F. Glasser

Anatomy and Neurobiology Department, Washington University School of Medicine,

St. Louis, MO 63110, USA

e-mail: vanessen@brainvis.wustl.edu 
methods include the systematic acquisition, analysis and sharing of large amounts of high-quality data through efforts such as the Human Connectome Project (HCP; Van Essen et al. 2012a, 2013). However, the indirect nature of in vivo imaging methods makes it critical to interpret results carefully and to seek better ways to compare and validate against 'ground truth' information available in animal models, particularly nonhuman primates such as the intensively studied macaque monkey. This chapter focuses on the functional organization and connectivity of human and macaque cerebral cortex. We consider (1) cortical organization, individual variability, and parcellation in both species; (2) connectivity in the macaque as revealed by tracers and by tractography; (3) connectivity of human cortex as revealed by noninvasive imaging methods; and (4) interspecies registration as a way to facilitate evolutionary comparisons and for cross-species connectivity validation.

\section{Cortical Cartography and Parcellation}

Useful Cortical Numbers It is informative to review a few basic anatomical facts about cortical organization, starting with some numbers related to the neocortex as a whole in macaques and humans. The cerebral neocortex is a sheet-like structure that in the macaque contains $\sim 1.4$ billion neurons/hemisphere deployed over a surface area of $\sim 105 \mathrm{~cm}^{2}$ per hemisphere-equivalent to a pair of $\sim 12 \mathrm{~cm}$ diameter cookies (Collins et al. 2010; Van Essen et al. 2012b). Human cortex has about fourfold more neurons ( $~ 8$ billion/hemisphere) and ninefold greater surface area ( $\sim 73 \pm 88 \mathrm{~cm}^{2} /$ hemisphere), equivalent to a pair of $35 \mathrm{~cm}$ pizzas (Azevedo et al. 2009; Van Essen et al. 2012c). The average number of neurons underneath each $\mathrm{mm}^{2}$ of the cortical surface is lower in humans $\left(8 \times 10^{3}\right)$ than in the macaque $\left(1.4 \times 10^{4}\right)$. Neuronal density is even lower in humans because the neocortex is on average slightly thicker in humans $(2.44 \mathrm{~mm}$, from Van Essen et al. 2012c; $2.68 \pm 0.40 \mathrm{~mm}$ for $196 \mathrm{HCP}$ subjects-Glasser et al. 2013b) than in macaques (1.86 $\pm 0.40 \mathrm{~mm}$ from 19 macaques-Glasser et al. 2013b).

Convolutions and Folding Variability In lissencephalic species (e.g., mice and marmosets), the cerebral neocortex wraps smoothly around most of the underlying white matter and subcortical gray matter structures, with no excess surface area. In contrast, gyrencephalic species have a disproportionately large number of neocortical relative to subcortical neurons, owing to differential neuronal proliferation (Finlay and Darlington 1995). Convolutions arise because the resultant expansion of neocortical surface area exceeds that of the subcortical nuclei and white matter (Van Essen 2006); they keep the brain physically compact, allowing a large cortical surface area to fit inside a cranial vault of modest volume. The specific pattern of cortical convolutions is distinct for each species, as is the degree of individual variability. Macaque cerebral cortex contains a dozen major sulci, with little variability across individuals in the pattern of folding or in the relationship of 
cortical areas to these folds. In contrast, the ninefold larger human cortical sheet is far more convoluted, with many primary, secondary and tertiary folds. It is also far more variable in the pattern of convolutions and in the relation of areal boundaries to cortical folds (Amunts et al. 2007; Fischl et al. 2008; Van Essen et al. 2012c).

Figure 1 illustrates the variability of human cortical folding in six exemplar right hemispheres, shown on the left as 3D 'midthickness' surfaces and on the right as FreeSurfer 'sulc' (depth) maps on inflated surfaces. This figure makes two important additional points, arising because the six individuals represent three pairs of monozygotic (MZ) twins taken from the HCP dataset. By visual inspection, $\mathrm{MZ}$ twins differ markedly in their folding patterns; qualitatively, the within-pair differences (between columns) are comparable to those between unrelated individuals (between rows). For example, the inferior frontal sulcus (IFS, red arrows) and posterior inferior temporal sulcus (pITS, yellow arrows) differ between MZ twins in the extent, depth, and relationship to nearby sulci. Quantitatively, however, the correlation of folding patterns in $\mathrm{MZ}$ twins is actually greater than that for unrelated individuals, as shown by K. Botteron, D. Dierker, D. Van Essen, R. Todd (2008 OHBM abstract and unpublished observations) and confirmed in the HCP dataset (Van Essen et al. 2014b). Moreover, the correlations are greater for MZ twins compared to dizygotic (DZ) twins, implying that there is significant heritability of folding patterns, though it is modest in magnitude (Van Essen et al. 2014b).

Developmental Considerations Given the complexity and variability of cortical convolutions, it is useful to briefly consider the underlying developmental events that provide useful mechanistic insights. Cortical folding takes place mainly in late prenatal development (the third trimester in humans) and has been suggested to be driven by mechanical tension along long-distance axons within the white matter

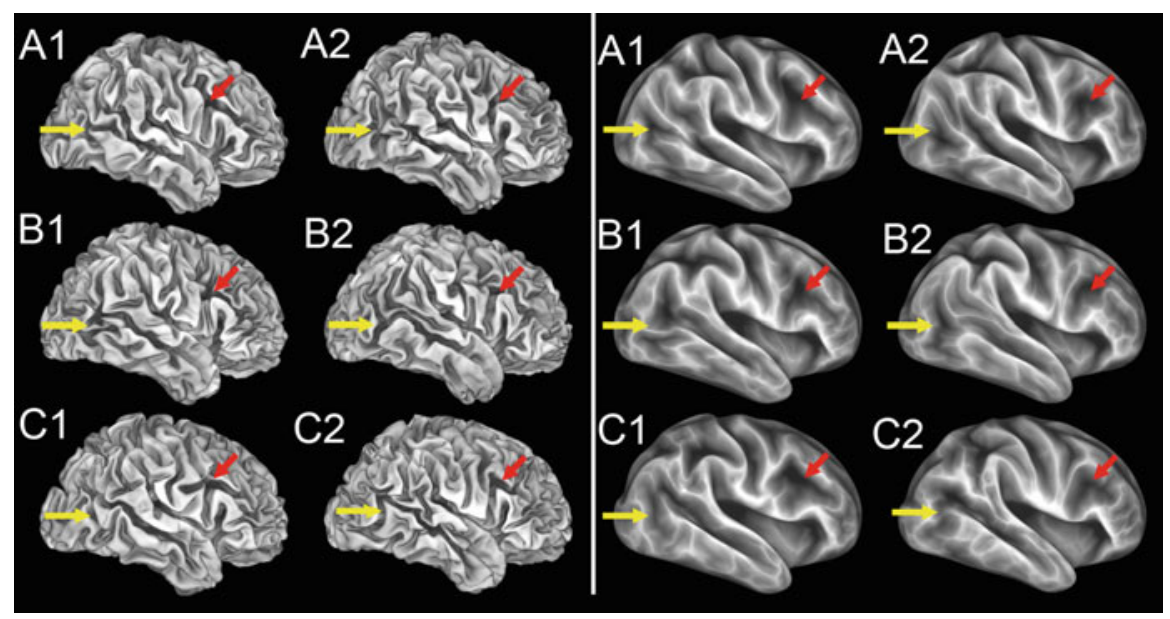

Fig. 1 Folding variability in human cortex is pronounced, even in identical twins. Left panels: three pairs of identical (MZ) twin pairs (A1-2, B1-2, C1-2), shown on midthickness surfaces. Right panels: corresponding inflated surfaces displaying FreeSurfer 'sulc' maps 
(Van Essen 1997). If the hypothesis of tension-based folding is correct, folding variability should largely reflect individual differences in connectivity, which in turn should reflect differences in the size as well as the connectivity profile of each cortical area. Consistent folding (e.g., the central sulcus) may arise from regions dominated by large areas and major pathways, whereas variable folding may reflect competition among smaller areas with greater connectional diversity. This hypothesis raises the intriguing puzzle of why cortical folding patterns differ so much in MZ twins. One possibility is that areal sizes and connectivity profiles are indeed markedly different in MZ twin pairs, owing to epigenetic and intrauterine environmental factors that impact cortical differentiation and wiring (in ways that are currently not understood). Alternatively, very different patterns of cortical folding in MZ twins may arise from subtle mechanical influences that 'buckle' or 'crumple' the cortex in ways that reflect stochastic fluctuations (e.g., external pressure on the cranium that affects overall brain shape) despite similar areal sizes and connectivity profiles. Careful analysis of the MZ and DZ twins in the HCP datasets may yield further insights that help address these issues.

Intersubject Alignment Irrespective of its developmental origins, folding variability poses important practical challenges whenever one aims to compare neuroimaging results obtained in different individuals. A widely used general strategy is to register individuals to a common spatial framework, i.e., an atlas. Intensive efforts have gone into improving brain atlases and the associated registration methods. For cerebral cortex, surface-based registration (SBR) and surface-based atlases have inherent advantages over conventional, volume-based registration because they respect the topology of the cortical sheet (Anticevic et al. 2008; Fischl et al. 1999; 2008; Frost and Goebel 2012; Tucholka et al. 2012; Van Essen et al. 2012c). Until recently, the available methods for SBR have used shape cues related to the folding pattern to constrain the registration from individuals to the atlas. This approach is inherently problematic for dealing with regions of high folding variability, especially since the location of the cortical areas and functionally specialized regions vary in relation to gyral and sulcal landmarks. Fortunately, new registration methods have recently emerged that capitalize on functionally relevant features (e.g., myelin maps, fMRI data) in conjunction with shape-based information (Sabuncu et al. 2010; Robinson et al. 2013). In the examples illustrated below, we capitalize on the Multimodal Surface Matching (MSM) method as applied to HCP and macaque datasets (Robinson et al. 2013, 2014; Glasser et al. 2014).

Regional Patterns Primate neocortex has a common overall architecture, but some features that vary systematically across the cortical sheet provide valuable guides to its functional organization. We first consider regional differences in myelin content, neuronal density, and dendritic arbor size, which collectively reflect important overall patterns that are independent of any particular parcellation scheme. Myelin content within the cortical ribbon can be estimated by taking the ratio between T1-weighted and T2-weighted structural MRI scans at each voxel and mapping this ratio onto the cortical surface (Glasser and Van Essen 2011). Figure 2 illustrates population-average maps of myelin content, displayed on inflated atlas surfaces of 


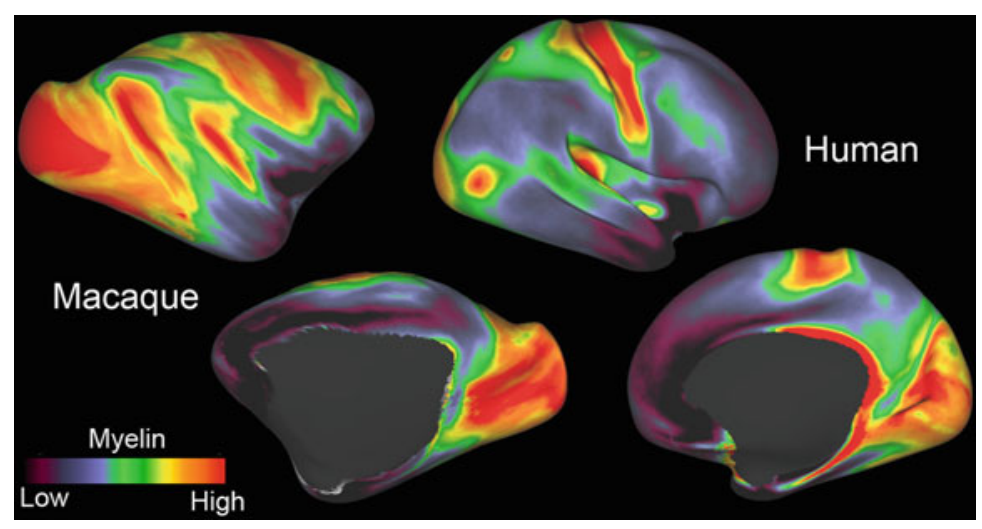

Fig. 2 Group-average myelin maps for macaque $(n=19$, Yerkes19) and human $(n=196, H C P)$, adapted from Glasser et al. (2013a, 2014). Myelin maps were generated by computing the $\mathrm{T} 1 \mathrm{w} / \mathrm{T} 2 \mathrm{w}$ ratio for each cortical gray matter voxel, mapping it to individual cortical surfaces, and registering the individuals to a species-specific atlas surface (Glasser and Van Essen 2011) using the MSM registration method (Robinson et al. 2013, 2014)

the macaque and human right hemispheres (Glasser et al. 2013b). Several important commonalities in the overall pattern for the two species are readily discernible. Most notably, early sensory and motor areas are heavily myelinated (red, yellow), whereas lightly myelinated regions (blue, indigo) occur mainly in regions of lateral temporal, parietal, and prefrontal cortex that are generally associated with higher cognitive functions. The most striking species difference is that the proportion of lightly myelinated cortex in humans far exceeds that in the macaque. This finding provides important clues about evolutionary expansion that are considered in a later section.

In the macaque, a fivefold range of neuronal cell density (neurons per gram of cortex) has been demonstrated using quantitative methods of cell fractionation applied to small patches of cortical gray matter (Collins et al. 2010). These cell density differences are correlated with the pattern of myelination, insofar as high neuronal density occurs in heavily myelinated early sensory and low density in the lightly myelinated 'higher' cortical areas (though the correlation is imperfect as heavily myelinated motor cortex has low neuronal density). Another important correlation is with dendritic arbor sizes (basal dendritic area) and spine numbers, determined by labeling individual neurons in lightly fixed tissue sampled from different cortical regions. Average dendritic arbor area and number of spines per neuron are low in areas V1 and V2 and are several-fold greater in temporal and prefrontal cortex (Elston 2000, 2002; Elston and Rosa 1997; Elston et al. 1999, 2001, 2006). In humans, comparable regional differences in dendritic arbor size and even larger differences in spine number have been demonstrated (Elston et al. 2001, 2006). There are also interesting correlations with patterns of cortical development. Human cortical surface area expands threefold after birth, but this postnatal expansion is nonuniform. The largest expansion ( 4-fold) occurs mainly in the lightly 
myelinated higher cognitive regions, whereas the least expansion ( 2-fold) occurs in early sensory areas (Hill et al. 2010). A likely cellular-level correlate is that, in the macaque, dendritic arbors sizes and synapse number increase between birth and adulthood in inferotemporal cortex, whereas there is a net decrease in both measures for early visual areas (Elston et al. 2010). Thus, in both species regional differences in neuronal density, average dendritic arbor size, and myelin content conform to a general pattern that provides important insights for understanding brain function, evolution, and development.

Cortical Parcellation Over the past century, a growing arsenal of methods has been used to parcellate cerebral cortex - that is, to identify distinct cortical areas based on differences across one or more four broad domains: architecture (cyto-, myelo- chemo-, and immunocyto-architectonics), connectivity (tracers and/or imaging-based), topography (e.g., retinotopy, somatotopy), and function (based on neurophysiology, lesions, and neuroimaging). Arguably the most accurate parcellation of mammalian cortex to date is in the lissencephalic mouse, where there is strong evidence for about 40 cortical areas based on a multi-modal analysis of architectonics, topography, and connectivity (Wang et al. 2012; see Burkhalter 2015). For macaque and human cortex, a consensus parcellation has yet to be attained in either species despite intensive efforts, because the differences between adjacent cortical areas are often subtle, are not always in concordance across different measures, and are susceptible to a variety of neurobiological confounds (e.g., differences in areal size across individuals, and in areal location relative to cortical folds) and other methodological confounds. Figure 3 illustrates composite parcellation schemes for macaque and human cortex based on studies that mapped

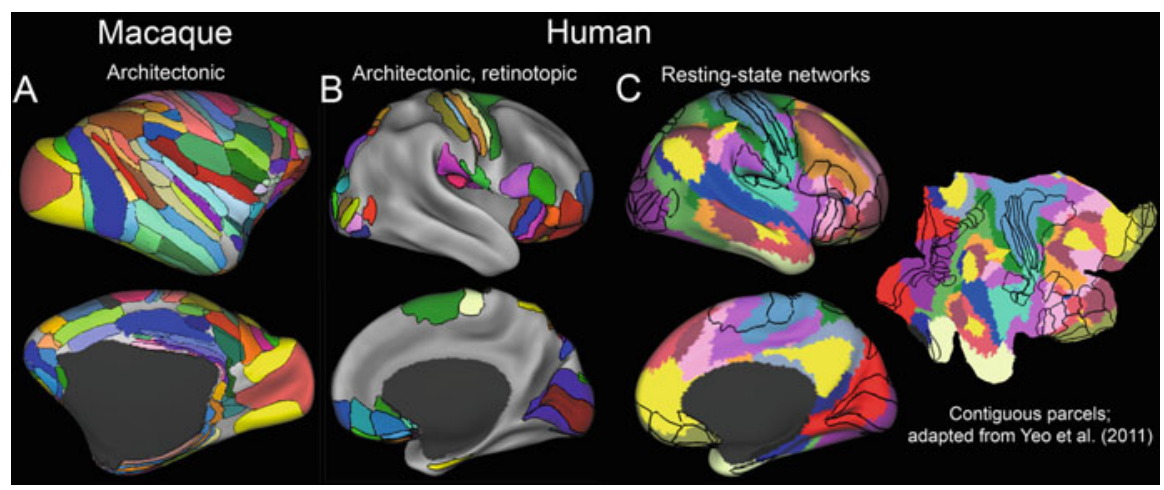

Fig. 3 Parcellations of macaque and human cortex. (a) A composite parcellation of macaque cortex into 129 areas based on published architectonic maps [adapted, with permission, from Van Essen et al. (2012b)]. (b) A 52-area parcellation of human cortex that is a composite of five published parcellations [adapted, with permission, from Van Essen et al. (2012c)]. (c) Restingstate network (RSN) parcels, from splitting Yeo et al.'s 17 networks into spatially contiguous subregions at least $50 \mathrm{~mm}^{2}$ in surface area. Areal boundaries from panel (b) are superimposed for comparison on inflated and flatmap surfaces 
parcels to the cortical surface accurately in individual subjects and then from individuals to an atlas surface using surface-based registration.

The macaque parcellation (Fig. 3a), based on a composite drawn from three architectonic analyses, includes 129 areas covering $93 \%$ of neocortex (Van Essen et al. 2012b). The size (surface area) of different areas spans a 100-fold range, and across individuals the size of any given area varies twofold or more. Comparisons across 15 published parcellation schemes registered to the atlas surface reveal a consensus for only a minority of these areas. For example, retinotopic mapping studies reveal a finer-grained parcellation of extrastriate visual cortex than in the above architectonic scheme (Kolster et al. 2009), but a consensus among parcellations based on different modalities has yet to be attained in the macaque for visual cortex beyond V1-V4 and MT (Van Essen et al. 2012b). Moreover, there is strong evidence for 'patch-like' functional specializations within some higher cortical regions. For example, lateral occipital and temporal cortex contains patches associated with processing of faces (Tsao et al. 2008) and color (Lafer-Sousa and Conway 2013), with each color patch just ventral to one of the face patches. An important but unresolved question is whether face and color patches should each be considered a distinct cortical 'area.' A plausible alternative is to consider them modular components of larger areas that are heterogeneous in function and connectivity, analogous to the modularity identified in areas V1, V2, and V4 (Deyoe et al. 1990). Reconciliation of the many discrepancies among extant macaque cortical parcellations will require additional data from multiple modalities that are acquired and analyzed with careful attention to spatial fidelity at every stage, including accurate intersubject registration to a surface-based atlas.

For human cortex, analyses based on accurate surface maps of architectonic and retinotopic areas have enabled identification of 52 areas, encompassing about one-third of the cortical sheet, to be mapped to a surface-based atlas (Fig. 3b; Van Essen et al. 2012c). If the intervening regions contain areas that are similar or slightly smaller in average surface area, as suggested by architectonic analyses yet to be accurately surface-mapped (Amunts et al. 2007), human cortex may contain 150-200 distinct areas in each hemisphere.

A valuable noninvasive approach to parcellating the entire cerebral cortex makes use of resting-state functional connectivity. 'Resting-state networks' (RSNs) represent regions that have similar fMRI (BOLD) time courses, as revealed using independent components analysis (ICA) or other ways of analyzing fMRI timeseries data. Importantly, many RSN components ('nodes') include spatially noncontiguous domains that can be widely dispersed (e.g., in different cortical lobes), reflecting a spatially distributed arrangement of regions that share similar fMRI time courses and are presumed to be involved in similar functions. To facilitate comparisons with spatially contiguous cortical areas derived using other methods (e.g., the architectonic and retinotopic areas in Fig. 3a, b), it is useful to subdivide each RSN into parcels that include only contiguous subregions. For example, Fig. 3c shows the 17 RSNs identified by Yeo et al. (2011) after splitting into a total of about 50 contiguous RSN parcels per hemisphere, displayed on a flatmap as well as inflated surfaces. Comparison of these RSN parcels with the 
overlaid boundaries of architectonic/retinotopic areas (from Fig. 3b) reveals numerous mismatches, because RSN parcel boundaries generally do not align with cortical areas defined by architectonic and topographic criteria. For example, the complex of early somatomotor areas (architectonic areas 1, 3a, 3b, and 4) includes two large RSN parcels that are split on the basis of somatotopy rather than areal boundaries. The cyan RSN parcel includes the face representation of somatomotor cortex but also extends into much of auditory cortex. The blue RSN parcel covers the body and limb representations but, except for the posterior boundary of area 2 (yellow arrow), the architectonic and RSN boundaries are not co-aligned. The separation into face vs. body RSN components is consistent with tracer studies in the macaque indicating differential anatomical connectivity patterns relating to somatotopy (Johnson et al. 1996; Matelli et al. 1998; Tanne-Gariepy et al. 2002; Luppino et al. 2003). The visual cortex includes three RSN components. One (in red) represents central vision of V1 and V2, plus many third-tier and fourthtier retinotopic areas and additional portions of ventral occipito-temporal cortex. A second (purple) represents peripheral V1 and V2 and other medial regions that emphasize peripheral vision. The third (light green) includes the 'IPS complex' of parietal visual areas but extends as a narrow strip laterally and ventrally.

Both neurobiological and methodological factors contribute to the mismatches between RSN-based and architectonic/retinotopic-based parcellation boundaries. Genuinely heterogeneous connectivity patterns within well-defined cortical areas are likely to be a major contributor, for reasons already noted above. Methodological factors include (1) inaccurate mapping to the cortical surface arising from cross-sulcal bleeding and mapping of signals from draining veins rather than cortical gray matter, both of which are exacerbated by larger voxel sizes (e.g., 3-mm isotropic voxels in Yeo et al. 2011); (2) noisy data arising from shortduration fMRI scans and imperfect denoising; and (3) inaccurate intersubject alignment when generating a population-average parcellation.

Prospects for improved human cortical parcellations are excellent, particularly in view of the high-quality data made available by the HCP (Van Essen et al. 2013; Smith et al. 2013). Of particular relevance are (1) more accurate cortical segmentation and surface reconstructions (higher resolution, improved algorithms; Glasser et al. 2013a); (2) higher quality rfMRI scans (1-h duration, multiband acquisition enabling $2 \mathrm{~mm}$ spatial and $0.7 \mathrm{~s}$ temporal resolution); (3) improved intersubject alignment using the aforementioned MSM algorithm (Robinson et al. 2014); plus additional methods refinements (Glasser et al. 2014). Even with these advances, there are significant challenges to improving the fidelity of cortical parcellation. For example, independent component analysis (ICA) can be used to generate 'soft' parcellations (each gray ordinate can have a weighted contribution to several network components), which can yield many ICA components that are substantially finer-grained than those illustrated in Fig. 3 (Van Essen et al. 2013; Smith et al. 2013). However, for reasons already mentioned, it is challenging to identify cortical areas by relying on RSNs alone. An attractive alternative in general is to use multiple MRI modalities (rfMRI, tfMRI, myelin, and cortical thickness being the most informative) and to combine across modalities in ways that maximize the 
prospect of identifying neurobiologically well-defined areas and sub-areas. Given the growing body of freely available HCP data, combined with ongoing methods refinements (especially in multimodal registration), high quality group-average human cortical parcellations are anticipated in the near future.

\section{Distributed Cortical Connectivity}

Our understanding of general principles of cortico-cortical connectivity has evolved dramatically in recent decades. Early studies suggested that each cortical area received only a few inputs and outputs (Van Essen 1979). Subsequent analyses using more sensitive tracers revealed evidence for hundreds of pathways among dozens of visual areas (Felleman and Van Essen 1991). There was also a growing realization that different pathways ranged widely in strength. Accurate quantitation of connection strengths and mapping to a surface-based parcellation is feasible (Lewis and Van Essen 2000a, b; Van Essen et al. 2001) but is tedious and technically demanding. Major progress on this front has come from a recent systematic effort from the laboratory of Henry Kennedy, revealing that connectivity profiles are more highly distributed and that connection strengths span a much wider range than previously realized (Knoblauch et al. 2015). Using a 91-area cortical parcellation and retrograde tracers injected into 29 cortical areas, Markov et al. (2012) determined that each cortical area receives on average inputs from 55 other areas out of a (minimum) 26 and (maximum) 87; when expressed as the fraction of retrogradely labeled neurons, these pathways vary over five orders of magnitude in connection strength (Markov et al. 2011, 2012; Knoblauch et al. 2015). This translates to 1615 inter-areal pathways out of 2610 possible in a $29 \times 91$ connectivity matrix. Most pathways are reciprocal and comparable in strength for the forward and feedback components, though the incidence of unidirectional pathways is greater than previously suspected (Markov et al. 2012).

These findings demonstrate that cortical connectivity is far from sparse, or 'small world,' when expressed as area-to-area connectivity. On the other hand, connections are much sparser when evaluated at finer-grained levels such as those of individual neurons or small patches of cortex. For example, each cortical neuron receives and makes about $10^{4}$ synapses on average; given $10^{9}$ neurons in the macaque, the upper bound of direct contacts is about $\sim 1$ neuron in $10^{5}$ (and even lower for human cortex). At an intermediate level of granularity, it is of interest to estimate the spatial extent of cortex that provides some degree of direct input to any given patch of cortex. Each $\mathrm{mm}^{2}$ patch of macaque cortex contains $\sim 10^{5}$ neurons and may contain $\sim 10^{9}$ synapses (but with significant regional variability, as noted above). An estimated $80 \%$ of inputs come from local (intra-areal) circuits, within a radius of several mm of a given patch (Markov et al. 2011). The remaining $20 \%$ of long-distance within-hemisphere inputs are distributed across the $10,000-\mathrm{mm}^{2}$ surface area of each hemisphere. Inspection of the long-distance retrogradely labeled neurons seen in histological sections (Markov et al. 2012, supplemental 
material) suggests that perhaps $10-20 \%$ of these patches contain at least one labeled neuron (but often many) and thus provide direct input to any given tracer injection site (Van Essen 2013). It would be possible to estimate this more accurately by mapping retrogradely labeled neurons directly from individual sections onto surface reconstructions (of individual hemispheres or directly to a surfacebased atlas). In any event, the density or sparseness of cortico-cortical connectivity depends greatly on the scale of analysis.

Similar principles of connectivity have recently been demonstrated in the mouse, based on an inter-areal parcellated connectome using anterograde tracers in ten areas of visual cortex (Wang et al. 2012; Burkhalter 2015) and on a mesoconnectome regional analysis involving nearly 300 bidirectionally analyzed pathways (Oh et al. 2014). As in the macaque, connection patterns in the mouse are highly distributed, and connection strengths vary over many orders of magnitude, suggesting that these principles may reflect a common mammalian plan.

Tracers vs. Tractography in the Macaque The aforementioned quantitative tracer studies in the macaque provide the closest currently available to ground truth connectivity data and are invaluable for assessing the performance of more indirect methods for estimating connectivity. For example, we have used this approach to evaluate diffusion imaging (dMRI) and probabilistic tractography analyzed in a postmortem macaque brain. This is especially important because tractography shows a strong 'gyral bias,' in which streamlines tend to terminate on gyral crowns, both in human and macaque cortex. This bias has been suggested to reflect actual anatomical connectivity (Nie et al. 2012). However, we contend that it conflicts with ground-truth neuroanatomy and instead reflects the tendency for the dominant fiber orientation in white matter 'blades' to be oriented along the axis pointing towards the gyral crown (Van Essen et al. 2014a). In myelin-stained sections, fibers 'peel off' to provide axons to and from the sulcal banks, but their radius of curvature is typically smaller than the dMRI voxel size (Van Essen et al. 2014a).

Figure $4 \mathrm{~b}$ shows that area $7 \mathrm{a}$ receives inputs from 60 of the 90 other areas shown in the Fig. 4a parcellation, and that connection strengths vary over $>5$ orders of magnitude, indicated by the logarithmic scale. Figure 4c shows the average connection strength (averaged across the two directions) for the subset of 29 areas that were also injection sites. For the tractography-based 'structural connectivity' and the same subset of areas (Fig. 4d), the strength of connectivity shows many similarities but also many differences, e.g., the pathway between area $\mathrm{PBr}$ is stronger for tractography than for tracers (arrows). A scatterplot of the pathway strength by the two methods for all connection pairs in the $29 \times 29$ matrix (Fig. 4e) shows a correlation that is significant for only the top $20 \%$ of tracer pathways (right of the vertical blue line).

Functional Connectivity Validation in the Macaque It is widely appreciated that functional connectivity is an inherently indirect surrogate for direct anatomical connectivity, because correlated activity can arise from common inputs and/or indirect anatomical pathways as well as direct connections (Smith et al. 2013). Hence, an important empirical question is the degree to which estimates of functional 


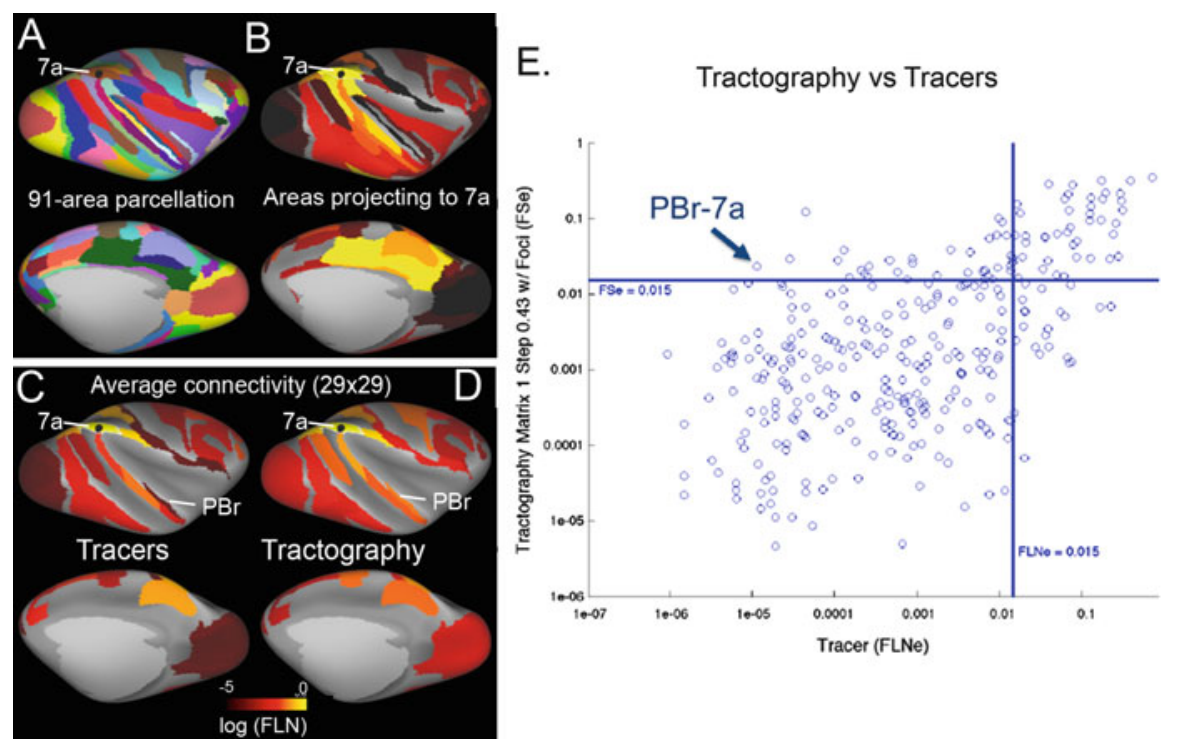

Fig. 4 (a) 91-area parcellation of macaque cortex on the inflated atlas surface. (b) Anatomical connectivity of area 7a based on a retrograde tracer injection into area 7a and quantitative mapping of its connection strength with all other areas (Markov et al. 2012). Connection strengths are on a $\log 10$ scale and span five orders of magnitude [same scale as for panels (c, d)]. (c) Average bidirectional tracer-based connectivity of area 7a based on the $29 \times 29$ 'edge-complete' bidirectional connectivity matrix. (d) Tractography-based connectivity, also for area 7 a but from a different macaque (cf., Van Essen et al. 2014a). Arrows in (c, d) point to area PBr, whose estimated connectivity based on tractography exceeds that determined using tracers. (e) Scatterplot of tracers-vs.-tractography connection strengths

connectivity correlate with anatomical connectivity in the macaque. Vincent et al. (2007) showed qualitatively that functional connectivity in the anesthetized macaque correlated well with anatomical connectivity when testing a seed region in the vicinity of area LIPv, whereas a seed region centered in area V1 revealed evidence of a combination of direct connections (e.g., with area MT) and indirect connections (e.g., with the horizontal meridian of contralateral area V1, which has no direct interhemispheric connections). A recent study showed a modest $(r=0.35)$ but highly significant correlation between functional connectivity in the anesthetized macaque and the Markov et al. (2012) quantitative parcellated connectivity matrix (Miranda-Dominguez et al. 2014). Importantly, the analysis revealed many false negatives (negative functional connectivity, or anti-correlation between areas that are strongly connected anatomically). Thus, as with tractography, it is important to remain mindful of the indirect nature of functional connectivity analyses and the limited capability for estimating actual connection strengths in nonhuman primates when using standard data acquisition and analysis methods.

Human Cortical Connectivity Structural connectivity (based on dMRI and tractography) and functional connectivity (based on resting-state fMRI) have 
already been discussed in relation to the macaque and also in relation to cortical parcellation in humans. However, much of the interest in these approaches derives from the prospect of charting the actual 'wiring diagram' of the human brain, how it varies across individuals, and how it relates to and contributes to individual differences in behavior. Indeed, this is the central focus of the HCP, as discussed extensively elsewhere (Van Essen et al. 2013; Smith et al. 2013). Here, we focus on several key observations and issues about the opportunities and challenges associated with this endeavor.

We focus on data and initial results from the HCP, as this represents the state-ofthe-art in acquiring and analyzing dMRI and rfMRI data in humans. Figure 5 shows a direct comparison between structural and functional connectivity in an individual HCP subject, using a seed location in the middle temporal gyrus (MTG, large green sphere). Structural connectivity based on probabilistic tractography (Fig. 5a) provides evidence of connectivity between the MTG and many regions, both nearby, at intermediate distances (two large patches in and near the angular gyrus, smaller green dots), and at long distances (e.g., on the inferior frontal gyrus). For the same seed location on the MTG (Fig. 5b), the map of functional connectivity shows many similarities but also many differences. The similarities indicate cross-modal consistency, suggesting that both patterns include many neurobiologically valid connections. For reasons already stated, differences may reflect neurobiological factors but also methodological biases or artifacts. For example, regions with strong functional connectivity but weak structural connectivity might in theory (white arrow in prefrontal cortex) reflect a pathway with only weak direct connections but strong indirect (polysynaptic) connections. Alternatively, a robust direct pathway may exist but fail to be captured by tractography owing to methodological limitations (e.g., inaccurate charting of the complex trajectories of fibers in white matter). Regions that suggest structural connectivity but not functional connectivity (e.g., red arrow in insular cortex) might reflect false positives in the tractography analysis

Despite these caveats and limitations, it is important to appreciate that the HCP structural and functional connectivity data represent major advances in our ability to acquire and analyze information about human brain circuitry. Examining

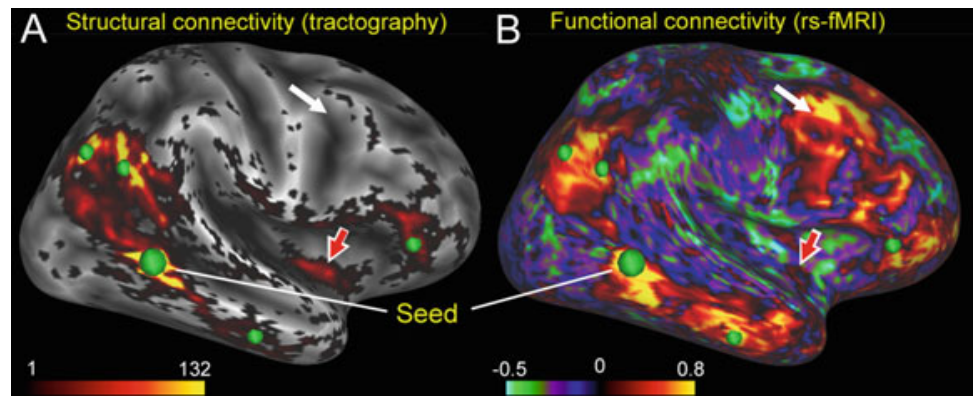

Fig. 5 Comparing structural connectivity (a) and functional connectivity (b) in an individual HCP subject for a seed vertex on the middle temporal gyrus [reproduced, with permission, from Van Essen et al. (2014a)] 
seed-based connectivity in individual subjects reveals that MRI-based data are inherently noisy and subject to bias. To gain meaningful insights, it is necessary to integrate across space (i.e., to use parcel-based analyses) and/or across the population (i.e., to capitalize on the large and growing number of HCP subjects). The need for parcel-based analysis that is based on functionally distinct brain subdivisions is a strong driver for the ongoing effort to improve human cortical parcellation using multimodal analysis, as discussed in an earlier section. The need for group-based analyses is a strong driver for the ongoing effort to improve intersubject alignment. Progress on both of these fronts will enhance the utility and interpretability of the HCP datasets and also other datasets that capitalize on these methodological improvements.

\section{Interspecies Registration}

Figures 2 and 3 provided evidence of many similarities in the functional organization of macaque and human cortex but also for major species differences in the relative sizes of different areas and regions presumed to be homologous. Interspecies surface-based registration offers a useful approach to comparing across species in ways that are systematic, quantitative, and objective; it will enable the use of tracer-based connectivity data in the macaque to inform and validate structural and functional connectivity findings in humans.

We have previously used landmark-constrained registration using about two dozen landmark contours that represent areal boundaries or other functional transitions presumed to reflect evolutionary homologies (Denys et al. 2004; Van Essen 2005). This approach provides evidence of hotspots of human evolution in lateral temporal, parietal, and prefrontal cortex that have expanded dramatically in the human lineage relative to that in the macaque; the pattern is remarkably similar to human postnatal cortical expansion, between birth and adulthood (Van Essen and Dierker 2007; Hill et al. 2010). To better understand this pattern of evolutionary divergence, it is desirable to have a larger set of known or presumed homologies between specie, and also to have improved methods of interspecies registration that handle the highly nonuniform spatial patterns of expansion. Here, we illustrate a brief progress report in this direction. It includes a more extensive set of landmark contours (38 in all), including orbitofrontal, lateral temporal, and parietal regions suspected to reflect homologies (Fig. 6a). Another advance involves an improved landmark-based registration method (the 'LVD' algorithm; Van Essen et al. 2012b) relative to an earlier method. The resultant map of interspecies cortical expansion (Fig. 6b) suggests that cortical expansion in these hotspots exceeds 30-fold, compared to the twofold to fourfold expansion over most of early visual cortex. Refinements to this general picture can be anticipated by invoking additional features to constrain interspecies registration, along with improved registration algorithms such as the multimodal surface matching method (Robinson et al. 2014) that can use continuously varying features (e.g., the myelin maps 


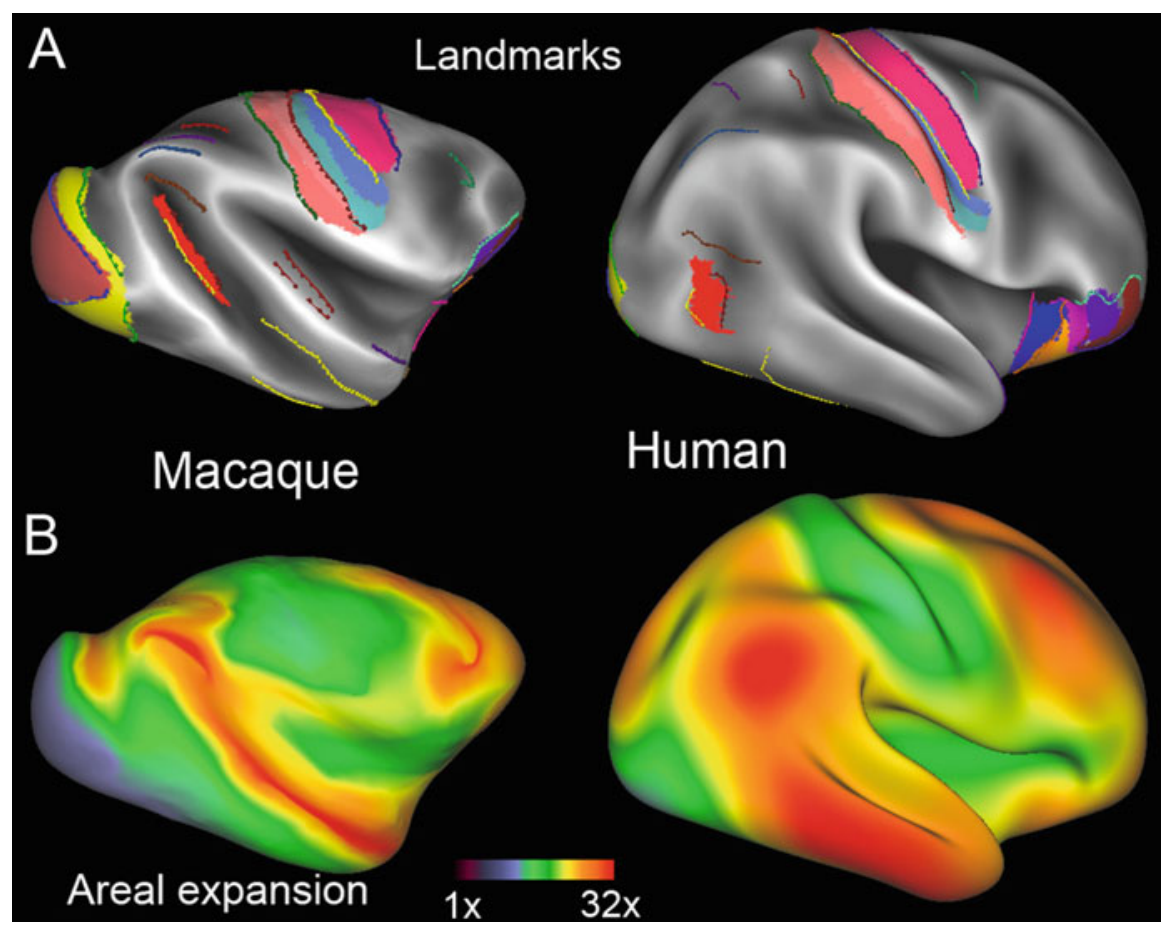

Fig. 6 (a) Landmarks used to register macaque to human cortex. (b) Areal expansion maps between human and macaque cortex, revealing hotspots of cortical expansion in lateral temporal, parietal, and frontal cortex. The expansion map was smoothed after initial computation of the interspecies area ratio to reduce neurobiologically implausible local irregularities

shown in Fig. 2) as well as discrete areal delimitations, rather than the purely landmark-constrained approach used to date. This will also be aided by incorporating chimpanzee cortex (Glasser et al. 2013b) as an important intermediary for examining evolutionary relationships.

It is now feasible to use interspecies registration as a test bed for evaluating human connectivity. Indeed, such an analysis was recently reported by MirandaDominguez et al. (2014), who used an earlier interspecies registration to compare human functional connectivity with the macaque tracer-based connectivity after registration to human cortex. They found a slightly higher correlation between human functional connectivity and interspecies-registered macaque connectivity $(r=0.42)$ than for the aforementioned macaque functional-vs.-tracer correlation $(r=0.35)$, raising the possibility that the quality of the functional connectivity data might be better for their human vs. their (anesthetized) macaque. Future analyses along these lines can be applied to tractography, different functional connectivity datasets (including $\mathrm{HCP}$ ) and different ways of analyzing the structural and functional connectivity (e.g., with vs. without regression of mean-gray-timecourse; 
partial vs. full correlation), and other possible measures as well as improved interspecies registrations.

\section{Concluding Comments}

Two decades ago, hardly anything was known about long-distance pathways in the human brain, other than the general distribution of major fiber tracts discernible from classical blunt dissection studies (e.g., Gluhbegovic and Williams 1980). This problem was famously articulated by Crick and Jones (1993) just when fMRI was beginning to emerge and before diffusion imaging began. The explosion of methodological advances has been dramatic but so have the many challenges of carefully analyzing and interpreting these highly complex datasets.

Open Access This chapter is distributed under the terms of the Creative Commons AttributionNoncommercial 2.5 License (http://creativecommons.org/licenses/by-nc/2.5/) which permits any noncommercial use, distribution, and reproduction in any medium, provided the original author(s) and source are credited.

The images or other third party material in this chapter are included in the work's Creative Commons license, unless indicated otherwise in the credit line; if such material is not included in the work's Creative Commons license and the respective action is not permitted by statutory regulation, users will need to obtain permission from the license holder to duplicate, adapt or reproduce the material.

\section{References}

Amunts K, Armstrong E, Malikovic A, Homke L, Mohlberg H, Schleicher A, Zilles K (2007) Gender-specific left-right asymmetries in human visual cortex. J Neurosci 27:1356-1364

Anticevic A, Dierker DL, Gillespie SK, Repovs G, Csernansky JG, Van Essen DC, Barch DM (2008) Comparing surface-based and volume-based analyses of functional neuroimaging data in patients with schizophrenia. Neuroimage 41:835-848

Azevedo FA, Carvalho LR, Grinberg LT, Farfel JM, Ferretti RE, Leite RE, Jacob Filho W, Lent R, Herculano-Houzel S (2009) Equal numbers of neuronal and nonneuronal cells make the human brain an isometrically scaled-up primate brain. J Comp Neurol 513:532-541

Burkhalter A (2015) The network for intracortical communication in mouse visual cortex. In: Kennedy H, Van Essen DC, Christen Y (eds) Micro-, meso- and macro-connectomics of the brain. Springer, Heidelberg

Collins CE, Airey DC, Young NA, Leitch DB, Kaas JH (2010) Neuron densities vary across and within cortical areas in primates. Proc Natl Acad Sci USA 107:15927-15932

Crick F, Jones E (1993) Backwardness of human neuroanatomy. Nature 361:109-110

Denys K, Vanduffel W, Fize D, Nelissen K, Sawamura H, Georgieva S, Vogels R, Van Essen D, Orban GA (2004) Visual activation in prefrontal cortex is stronger in monkeys than in humans. J Cogn Neurosci 16:1505-1516

Deyoe EA, Hockfield S, Garren H, Van Essen DC (1990) Antibody labeling of functional subdivisions in visual cortex: cat-301 immunoreactivity in striate and extrastriate cortex of the macaque monkey. Vis Neurosci 5:67-81

Elston GN (2000) Pyramidal cells of the frontal lobe: all the more spinous to think with. J Neurosci 20:RC95

Elston GN (2002) Cortical heterogeneity: implications for visual processing and polysensory integration. J Neurocytol 31:317-335 
Elston GN, Rosa MG (1997) The occipitoparietal pathway of the macaque monkey: comparison of pyramidal cell morphology in layer III of functionally related cortical visual areas. Cereb Cortex 7:432-452

Elston GN, Tweedale R, Rosa MG (1999) Cortical integration in the visual system of the macaque monkey: large-scale morphological differences in the pyramidal neurons in the occipital, parietal and temporal lobes. Proc Biol Sci 266:1367-1374

Elston GN, Benavides-Piccione R, DeFelipe J (2001) The pyramidal cell in cognition: a comparative study in human and monkey. J Neurosci 21:163

Elston GN, Benavides-Piccione R, Elston A, Zietsch B, Defelipe J, Manger P, Casagrande V, Kaas JH (2006) Specializations of the granular prefrontal cortex of primates: implications for cognitive processing. Anat Rec A Discov Mol Cell Evol Biol 288:26-35

Elston GN, Oga T, Okamoto T, Fujita I (2010) Spinogenesis and pruning from early visual onset to adulthood: an intracellular injection study of layer III pyramidal cells in the ventral visual cortical pathway of the macaque monkey. Cereb Cortex 20:1398-1408

Felleman DJ, Van Essen DC (1991) Distributed hierarchical processing in the primate cerebral cortex. Cereb Cortex 1:1-47

Finlay BL, Darlington RB (1995) Linked regularities in the development and evolution of mammalian brains. Science 268:1578-1584

Fischl B, Sereno MI, Tootell RB, Dale AM (1999) High-resolution intersubject averaging and a coordinate system for the cortical surface. Hum Brain Mapp 8:272-284

Fischl B, Rajendran N, Busa E, Augustinack J, Hinds O, Yeo B, Mohlberg H, Amunts K, Zilles K (2008) Cortical folding patterns and predicting cytoarchitecture. Cereb Cortex 18:1973

Frost MA, Goebel R (2012) Measuring structural-functional correspondence: spatial variability of specialised brain regions after macro-anatomical alignment. Neuroimage 59:1369-1381

Glasser M, Van Essen DC (2011) Mapping human cortical areas in vivo based on myelin content as revealed by T1 and T2-weighted MRI. J Neurosci 31:11597-11616

Glasser MF, Sotiropoulos SN, Wilson JA, Coalson T, Fischl B, Andersson J, Xu J, Jbabdi S, Webster M, Polimeni J, Van Essen DC, Jenkinson M (2013a) The minimal preprocessing pipelines for the Human Connectome Projects. Neuroimage 80:105-124 (Special issue on mapping the Connectome)

Glasser MF, Goyal MS, Press TM, Raichle ME, Van Essen DC (2013b) Trends and properties of human cerebral cortex: correlations with cortical myelin content. Neuroimage 93 Pt 2: 165-175, (Special issue on In Vivo Brodmann Mapping). doi: 10.1016/n.neuroimage.2013. 03.060

Glasser MF, Goyal MS, Press TM, Raichle ME, Van Essen DC (2013c) Trends and properties of human cerebral cortex: correlations with cortical myelin content. Neuroimage $93 \mathrm{Pt} 2$ : 165-175, (Special issue on In Vivo Brodmann Mapping). doi: 10.1016/n.neuroimage.2013. 03.060

Gluhbegovic N, Williams TH (1980) The human brain: a photographic guide. HarperCollins, New York, p 176

Hill J, Inder T, Neil J, Dierker D, Harwell J, Van Essen D (2010) Similar patterns of cortical expansion during human development and evolution. Proc Natl Acad Sci USA 107: $13135-13140$

Johnson PB, Ferraina S, Bianchi L, Caminiti R (1996) Cortical networks for visual reaching: physiological and anatomical organization of frontal and parietal lobe arm regions. Cereb Cortex 6:102-119

Knoblauch K, Ercsey-Ravasz M, Kennedy H, Toroczkai Z et al (2015) The brain in space. In: Kennedy H, Van Essen DC, Christen Y (eds) Micro-, meso- and macro-connectomics of the brain. Springer, Heidelberg

Kolster H, Mandeville JB, Arsenault JT, Ekstrom LB, Wald LL, Vanduffel W (2009) Visual field map clusters in macaque extrastriate visual cortex. J Neurosci 29:7031-7039

Lafer-Sousa R, Conway BR (2013) Parallel, multi-stage processing of colors, faces and shapes in macaque inferior temporal cortex. Nat Neurosci 16:1870-1878 
Lewis J, Van Essen D (2000a) Corticocortical connections of visual, sensorimotor, and multimodal processing areas in the parietal lobe of the macaque monkey. J Comp Neurol 428: $112-137$

Lewis JW, Van Essen DC (2000b) Mapping of architectonic subdivisions in the macaque monkey, with emphasis on parieto-occipital cortex. J Comp Neurol 428:79-111

Luppino G, Rozzi S, Calzavara R, Matelli M (2003) Prefrontal and agranular cingulate projections to the dorsal premotor areas F2 and F7 in the macaque monkey. Eur J Neurosci 17:559-578

Markov NT, Misery P, Falchier A, Lamy C, Vezoli J, Quilodran R, Gariel MA, Giroud P, ErcseyRavasz M, Pilaz LJ, Huissoud C, Barone P, Dehay C, Toroczkai Z, Van Essen DC, Kennedy H, Knoblauch K (2011) Weight consistency specifies regularities of macaque cortical networks. Cereb Cortex 21:1254-1272

Markov NT, Ercsey-Ravasz MM, Ribeiro Gomes AR, Lamy C, Magrou L, Vezoli J, Misery P, Falchier A, Quilodran R, Gariel MA, Sallet J, Gamanut R, Huissoud C, Clavagnier S, Giroud P, Sappey-Marinier D, Barone P, Dehay C, Toroczkai Z, Knoblauch K, Van Essen DC, Kennedy H (2012) A weighted and directed interareal connectivity matrix for macaque cerebral cortex. Cereb Cortex 24(1):17-36, Epub 2012/09/27

Matelli M, Govoni P, Galletti C, Kutz DF, Luppino G (1998) Superior area 6 afferents from the superior parietal lobule in the macaque monkey. J Comp Neurol 402:327-352

Miranda-Dominguez O, Mills BD, Grayson D, Woodall A, Grant KA, Kroenke CD, Fair DA (2014) Bridging the gap between the human and macaque connectome: a quantitative comparison of global interspecies structure-function relationships and network topology. J Neurosci 34: $5552-5563$

Nie J, Guo L, Li K, Wang Y, Chen G, Li L, Chen H, Deng F, Jiang X, Zhang T, Huang L, Faraco C, Zhang D, Guo C, Yap PT, Hu X, Li G, Lv J, Yuan Y, Zhu D, Han J, Sabatinelli D, Zhao Q, Miller LS, Xu B, Shen P, Platt S, Shen D, Hu X, Liu T (2012) Axonal fiber terminations concentrate on gyri. Cereb Cortex 22:2831-2839

Oh SW, Harris JA, Ng L, Winslow B, Cain N, Mihalas S, Wang Q, Lau C, Kuan L, Henry AM, Mortrud MT, Ouellette B, Nguyen TN, Sorensen SA, Slaughterbeck CR, Wakeman W, Li Y, Feng D, Ho A, Nicholas E, Hirokawa KE, Bohn P, Joines KM, Peng H, Hawrylycz MJ, Phillips JW, Hohmann JG, Wohnoutka P, Gerfen CR, Koch C, Bernard A, Dang C, Jones AR, Zeng H (2014) A mesoscale connectome of the mouse brain. Nature 2014(508):207-214

Robinson E, Jbabdi S, Andersson J, Smith S, Glasser M, Van Essen D, Burgess G, Harms M, Barch D, Jenkinson M (2013) Multimodal surface matching: fast and generalisable cortical registration using discrete optimisation. Information processing in medical imaging. Springer, New York, pp 475-476

Robinson EC, Jbabdi S, Glasser MF, Andersson J, Burgess GC, Harms MP, Smith SM, Van Essen DC, Jenkinson M (2014) MSM: a new flexible framework for multimodal surface matching. Neuroimage 100:414-426

Sabuncu MR, Singer BD, Conroy B, Bryan RE, Ramadge PJ, Haxby JV (2010) Function-based intersubject alignment of human cortical anatomy. Cereb Cortex 20:130-140

Smith SM, Vidaurre D, Beckmann CF, Glasser MF, Jenkinson M, Miller KL, Nichols TE, Robinson EC, Salimi-Khorshidi G, Woolrich MW, Barch DM, Ugurbil K, Van Essen DC (2013) Functional connectomics from resting-state fMRI. Trends Cogn Sci 17:666-682

Tanne-Gariepy J, Rouiller EM, Boussaoud D (2002) Parietal inputs to dorsal versus ventral premotor areas in the macaque monkey: evidence for largely segregated visuomotor pathways. Exper Hirnforsch 145:91-103

Tsao DY, Moeller S, Freiwald WA (2008) Comparing face patch systems in macaques and humans. Proc Natl Acad Sci USA 105:19514-19519

Tucholka A, Fritsch V, Poline JB, Thirion B (2012) An empirical comparison of surface-based and volume-based group studies in neuroimaging. Neuroimage 63:1443-1453

Van Essen DC (1979) Visual areas of the mammalian cerebral cortex. Annu Rev Neurosci 2: $227-263$ 
Van Essen DC (1997) A tension-based theory of morphogenesis and compact wiring in the central nervous system. Nature 385:313-318

Van Essen DC (2005) A population-average, landmark- and surface-based (PALS) atlas of human cerebral cortex. Neuroimage 28:635-662

Van Essen DC (2006) Cerebral cortical folding patterns in primates: why they vary and what they signify. In: Kaas J (ed) Evolution of the nervous system. Academic, Oxford

Van Essen DC (2013) Cartography and connectomes. Neuron 80:775-790

Van Essen DC, Dierker DL (2007) Surface-based and probabilistic atlases of primate cerebral cortex. Neuron 56:209-225

Van Essen DC, Drury HA, Dickson J, Harwell J, Hanlon D, Anderson CH (2001) An integrated software suite for surface-based analyses of cerebral cortex. J Am Med Inform Assoc 8: 443-459

Van Essen DC, Ugurbil K, Auerbach E, Barch D, Behrens TE, Bucholz R, Chang A, Chen L, Corbetta M, Curtiss SW, Della Penna S, Feinberg D, Glasser MF, Harel N, Heath AC, LarsonPrior L, Marcus D, Michalareas G, Moeller S, Oostenveld R, Petersen SE, Prior F, Schlaggar BL, Smith SM, Snyder AZ, Xu J, Yacoub E (2012a) The human connectome project: a data acquisition perspective. Neuroimage 62:2222-2231

Van Essen DC, Glasser MF, Dierker D, Harwell J (2012b) Cortical parcellations of the macaque monkey analyzed on surface-based atlases. Cereb Cortex 22:2227-2240. doi:10.1093/ cercor/bhr2290

Van Essen DC, Glasser MF, Dierker D, Harwell J, Coalson T (2012c) Parcellations and hemispheric asymmetries of human cerebral cortex analyzed on surface-based atlases. Cereb Cortex 22:2241-2262. doi:10.1093/cercor/bhr2291

Van Essen DC, Smith S, Barch D, Behrens TEJ, Yacoub E, Ugurbil K (2013) The WU-Minn human connectome project: an overview. Neuroimage 62:1299-1310, Jan 10 [Epub ahead of print]

Van Essen DC, Jbabdi S, Sotiropoulos SN, Chen C, Dikranian K, Coalson T, Harwell J, Behrens TEJ, Glasser MF (2014a) Mapping connections in humans and nonhuman primates: aspirations and challenges for diffusion imaging. In: Johansen-Berg H, Behrens T (eds) Diffusion MRI, 2nd edn. Academic, San Diego, pp 337-358

Van Essen D, Glasser MF, Robinson E, Xu C, Jenkinson M, Dierker D, Nichols T, Smith S (2014b) Heritability of brain structure, function, and connectivity in human connectome project data. In: Annual meeting of the organization for human brain mapping, Hamburg, Germany, 8-12 June 2014

Vincent JL, Patel GH, Fox MD, Snyder AZ, Baker JT, Van Essen DC, Zempel JM, Snyder LH, Corbetta M, Raichle ME (2007) Intrinsic functional architecture in the anaesthetized monkey brain. Nature 447:83-86

Wang Q, Sporns O, Burkhalter A (2012) Network analysis of corticocortical connections reveals ventral and dorsal processing streams in mouse visual cortex. J Neurosci 32:4386-4399

Yeo BT, Krienen FM, Sepulcre J, Sabuncu MR, Lashkari D, Hollinshead M, Roffman JL, Smoller JW, Zollei L, Polimeni JR, Fischl B, Liu H, Buckner RL (2011) The organization of the human cerebral cortex estimated by intrinsic functional connectivity. J Neurophysiol 106:1125-1165 